\title{
Pesquisa e extensão no Instituto Federal Catarinense: Uma investigação sobre as problemáticas e uma revisão de posturas da gestão institucional
}

\author{
Research and extension in the Instituto Federal Catarinense: An \\ investigation into the issues and a review of institutional management's \\ attitudes
}

Recebido: 29/01/2019 | Revisado: 14/02/2020 | Aceito: 22/03/2020|

Publicado: $16 / 06 / 2021$

Marlon Cordeiro Domenech

ORCID: https://orcid.org/0000-0003-33252282

Instituto Federal Catarinense

E-mail: marlon.domenech@ifc.edu.br

Vanderlei Cristiano Juraski

ORCID: https://orcid.org/0000-0002-35114708

Instituto Federal Catarinense

E-mail: vanderlei.juraski@ifc.edu.br

Marcelo Volpatto Marques

ORCID: https://orcid.org/0000-0001-69580283

Instituto Federal Catarinense

E-mail: marcelo.marques@ifc.edu.br

Viviane Menes Da Silva

ORCID: https://orcid.org/0000-0002-1775-

3119

Instituto Federal Catarinense

E-mail: vmenesdasilva@gmail.com

Ana Luíza Pozzobom

ORCID: https://orcid.org/0000-0002-70784870

Instituto Federal Catarinense

E-mail: alpozzobom@gmail.com

Como citar:

DOMENECH, M. C.; JURASKI, V. C.; MARQUES, M. V.; SILVA, V. M.; POZZOBOM, A. L.; Pesquisa e extensão no Instituto Federal catarinense: Uma investigação sobre as problemáticas e uma revisão de posturas da gestão institucional. Revista Brasileira da Educação Profissional e Tecnológica, [S.I.], v. 1, n. 20 , p. 1-21, e10860, jun. 2021. ISSN 2447 1801.

This work is licensed under a $\underline{\text { Creative }}$ Commons Attribution 4.0 Unported License.

\section{Resumo}

Os Institutos Federais possuem, como parte de sua institucionalidade, um modelo de Educação que integra ensino, pesquisa e extensão ainda no Ensino Médio, como parte da Educação Profissional voltada à formação integral dos indivíduos. A partir disso, este trabalho foi motivado pela percepção, em um dos Campi do Instituto Federal Catarinense (IFC), de que pesquisa e extensão estavam pouco vinculadas à sociedade e eram pouco realizadas. Assim, este trabalho apresenta uma visão dos desafios para a realização de pesquisa e extensão no IFC, uma tentativa de entendê-los e propõe soluções a estes, do ponto de vista de coordenadores de projetos e gestores de pesquisa e extensão.

Palavras-Chave: Pesquisa; Extensão; Educação Profissional e Tecnológica. Instituto Federal.

\begin{abstract}
The Institutos Federais have, as part of its institutionality, an Education Model that integrates teaching, research and extension within High School, as part of a Professional Education aimed to the integral development of individuals. So, this work was motivated by the perception, in a Campus of Instituto Federal Catarinense (IFC), that research and extension activities were not well linked to society and were poorly performed. Therefore, this work presents a vision of the challenges for performing research and extension at IFC, an attempt to understand such challenges and propose them solutions, from the point of view of project coordinators and institutional managers of research and extension.
\end{abstract}

Keywords: Research; Extension; Technological and Professional Education; Instituto Federal. 


\section{INTRODUÇÃO}

A pesquisa científica tem sido, há muito tempo, um dos pilares do desenvolvimento da humanidade. Dentro do Instituto Federal Catarinense (IFC), no contexto da Educação Profissional e Tecnológica (EPT), tem-se a pesquisa nas formas da pesquisa aplicada, a pesquisa de base e o desenvolvimento tecnológico (FREITAS JUNIOR; CARVALHO, 2020). A visão é de que a pesquisa também é uma ferramenta para o desenvolvimento dos sujeitos envolvidos em relação ao processo de aprendizagem e o senso de colocação na sociedade como indivíduos ativos na transformação e melhoria social. A extensão, por sua vez, é uma das maneiras de levar à sociedade o conhecimento e as soluções produzidas nas atividades de pesquisa, bem como uma oportunidade de aproximar-se desta sociedade e entender suas demandas, as quais direcionam ações institucionais de ensino, pesquisa e extensão. Aliar pesquisa e extensão permite entender e responder às demandas sociais, enquanto se dá o processo educativo, sendo assim elementos que possibilitam a formação cidadã.

Apesar dessas possibilidades, realizar pesquisa e extensão no contexto da EPT de Nível Médio tem sido um desafio (MARQUES; VIEIRA, 2020). Este trabalho expressa uma visão de tais desafios, uma tentativa de entendê-los e propõe soluções a estes, do ponto de vista de atores que atuaram enquanto servidores do Campus Fraiburgo do IFC, coordenadores de projetos de pesquisa e extensão e, ao mesmo tempo, Coordenadores de Pesquisa, Pós-Graduação e Inovação e de Extensão, Estágios e Egressos deste Campus. Assim, este trabalho apresenta uma tentativa de entender a problemática e os desafios, usando de visões de outros servidores da instituição, bem como apresenta propostas de soluções, que foram formuladas com colaboração destes outros servidores mas tiveram, principalmente, um viés de "visão da gestão", proporcionado pela experiência dos autores enquanto gestores. Entendemos que essa visão dual, enquanto gestores e servidores que realizam atividades de pesquisa e extensão, permite entender de maneira singular os desafios que se apresentam e formular respostas a estes desafios, alinhadas à gestão e também alinhadas aos servidores que, de fato, realizam tais atividades.

O Campus em questão possui em torno de 60 servidores, sendo aproximadamente metade docentes e metade técnicos-administrativos em educação (TAEs). São oferecidos os cursos de Técnico em Informática Integrado ao Ensino Médio, Técnico em Segurança do Trabalho Subsequente ao Ensino Médio, uma Especialização em Educação e, em 2018, iniciou-se a oferta de um Tecnólogo em Análise e Desenvolvimento de Sistemas. São também ofertados diversos cursos de formação inicial e continuada na modalidade de Qualificação Profissional, de oferta não-regular.

O problema que motivou a realização deste trabalho foi a percepção, dentro do Campus, de que ações de pesquisa e extensão estavam pouco vinculadas à sociedade e eram realizadas em pequena quantidade e quase que exclusivamente pelas mesmas pessoas. Como pesquisa e extensão não estavam curricularizadas à época da realização deste trabalho (2018), tais ações limitavam-se aos projetos fomentados pelo Campus e pela Reitoria em editais de bolsas estudantis. Como exemplo, entre 2016 e 2018, todos os editais de bolsas estudantis de pesquisa do Campus tiveram um número igual ou menor de propostas em relação à quantidade de bolsas ofertadas. Já os editais de extensão, em apenas 1 edital houve mais projetos 
propostos do que bolsas oferecidas. A participação nos editais da Reitoria também limita-se a um ou dois projetos por ano, no período entre 2016 e 2018.

A partir dessa problemática, buscou-se atender aos seguintes objetivos com este trabalho:

1. Buscar parcerias entre o IFC e instituições públicas e privadas nas áreas de pesquisa, desenvolvimento tecnológico e extensão;

2. Propor iniciativas que tenham a perspectiva de integrar ensino, pesquisa e extensão; e

3. Entender como melhor fomentar as atividades de pesquisa e extensão do IFC Campus Fraiburgo.

O restante deste trabalho está organizado da seguinte maneira. A Seção 2 apresenta a metodologia aplicada para atingir cada um dos objetivos. Em seguida, são apresentados os resultados e uma discussão sobre estes na Seção 4, seguido das conclusões e considerações finais na Seção 5 .

\section{METODOLOGIA}

Para atingir o primeiro objetivo (Buscar parcerias entre o IFC e instituições públicas e privadas nas áreas de pesquisa, desenvolvimento tecnológico e extensão) foram realizados os seguintes procedimentos metodológicos:

1. Levantamento de demandas/oportunidades de pesquisa e extensão entre servidores do IFC Campus Fraiburgo;

2. Elaboração de uma pesquisa para levantamento de oportunidades de pesquisa e extensão entre a comunidade externa ao IFC Campus Fraiburgo;

3. Aplicação de ambas as pesquisas; e

4. Tratamento e análise dos dados obtidos.

Referente ao procedimento 1, por entender que demandas de pesquisa existentes entre os servidores já tem possibilidade de tornarem-se projetos, por iniciativa do próprio servidor, os autores optaram por fazer um levantamento dos trabalhos de projeto integrador realizados pelos alunos do Ensino Médio Integrado (turmas de $2^{\circ}$ e $3^{\circ}$ ano) que teriam potencial de tornarem-se projetos de pesquisa ou de extensão. A disciplina de projeto integrador é composta de 3 etapas, cada uma realizada em um dos anos do ensino médio. Na primeira etapa, abordam-se aspectos metodológicos em relação à realização e concepção de projetos. Na segunda etapa, ocorre a escrita de uma proposta de projeto, enquanto na terceira etapa esta proposta é colocada em execução.

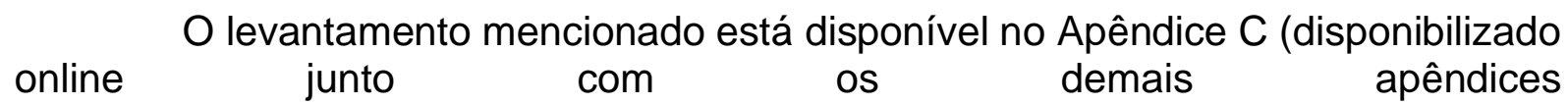
em https://drive.google.com/file/d/1iiQG7zay2byv TOKIGM2LXVxZ PE3WAQ/view? usp=sharing) e deve ser disponibilizado aos servidores e estudantes em 2019, visando incentivar a realização de projetos.

Em relação aos procedimentos 2, 3 e 4, a abordagem foi realizada em 4 etapas que aconteceram concomitantemente, a saber: 
- Colaboração com outras instituições para a criação do projeto de uma incubadora de empresas na cidade Fraiburgo, na qual o IFC Campus Fraiburgo está localizado, o que pode fomentar atividades de pesquisa e de extensão em parceria com o IFC. O projeto de lei resultante deste trabalho está disponível no sítio da prefeitura (FRAIBURGO, 2019).

- Envio de Ofício ao Gabinete do Prefeito de Fraiburgo, propondo o levantamento de problemas junto às Secretarias Municipais, os quais poderiam tornar-se projetos de pesquisa/extensão em parceria. O ofício está disponível no Apêndice D e aguarda resposta;

- Reuniões com a Associação Empresarial de Fraiburgo (ACIAF), buscando possibilidades de projetos em parceria. A partir disso, iniciaram-se as tratativas para formalizar uma parceria para desenvolvimento de um projeto de software para a área Agrícola, intitulado "Caderno de Campo". Foi apresentado um planejamento para execução da proposta, o qual está em análise pela referida Associação; e

- Tratativas com a empresa Tratativas com a empresa AGF (http://www.antigranizo.com.br/index.htm) para firmar parceria visando a digitalização/informatização da captura de dados do radar meteorológico e disponibilização de previsão do tempo imediata, para fins de combate ao granizo na região do Meio Oeste Catarinense. A demanda foi iniciada com o Campus Fraiburgo e assumida pelo Campus Luzerna (Curso de Engenharia de Controle e Automação).

Para atingir o segundo objetivo (Propor iniciativas que tenham a perspectiva de integrar ensino, pesquisa e extensão) procedeu-se à publicação do Edital 040/2017 Apresentação de projetos integrados de pesquisa e extensão e seleção de estudantes do ensino técnico como bolsistas do IFC Campus Fraiburgo (INSTITUTO FEDERAL CATARINENSE, 2017). Neste edital foi previsto que projetos aprovados teriam 2 cotas de bolsa (ao invés de 1, que tradicionalmente se concede) e deveriam integrar pesquisa e extensão enquanto concepção do projeto e em suas atividades. Também, realizou-se a III Edição da FEPEX - Feira de Ensino, Pesquisa e Extensão do IFC Campus

(https://publicacoes.ifc.edu.br/index.php/fepexfraiburgo/issue/view/24), 0 que caracteriza-se como uma ação que visou atender a este objetivo.

Para atingir o terceiro objetivo ( Entender como melhor fomentar as atividades de pesquisa e extensão do IFC Campus Fraiburgo) foram realizados os seguintes procedimentos metodológicos:

1. Elaboração de uma pesquisa de opinião a ser aplicada aos servidores do IFC Campus Fraiburgo;

2. Aplicação da pesquisa de opinião aos servidores;

3. Tratamento dos dados obtidos na pesquisa de opinião;

4. Elaboração de questionário a ser enviado a outros Campi da Rede Federal de Educação Profissional, Científica e Tecnológica, sobre práticas institucionais de fomento à pesquisa e fomento à integração com os arranjos produtivos, sociais e culturais locais;

5. Tratamento dos dados obtidos no questionário; e 
6. Análise das informações obtidas.

A pesquisa de opinião resultante do procedimento 1 está disponível no Apêndice $A$ e seus resultados estão disponíveis em: https://docs.google.com/spreadsheets/d/1DGXgG9OeDENYVz8uqb9qz9OW8dPsy8 NMqS_F_j_KUYw/edit?usp=sharing. Foi utilizado o Google Formulários como ferramenta de aplicação do questionário. A aplicação do questionário (procedimento 2) foi realizada entre $05 / 03 / 2018$ e 21/03/2018 via e-mail institucional. No conjunto de aproximadamente 60 servidores foram obtidas 17 respostas. $O$ tratamento dos dados (procedimento 3) foi realizado usando o Google Planilhas. Uma análise dos dados obtidos é apresentada na Seção 3.

A elaboração do questionário a ser enviado aos demais Campi foi feita com base nas respostas do questionário aplicado aos servidores do Campus Fraiburgo. Enquanto o primeiro teve perguntas com respostas descritivas, o segundo tinha perguntas com respostas de múltipla escolha, visando facilitar a análise posterior. $O$ questionário foi aplicado também usando o Google Formulários, entre as datas de 14/06/2018 e 29/06/2018. O envio aos servidores foi feito via Coordenação Geral de Comunicação (CECOM) da Reitoria do IFC, por meio do e-mail institucional. O questionário está disponível no Apêndice $\mathrm{B}$. As respostas estão disponíveis em dois formatos: https://docs.google.com/forms/d/1AacLR8aqdTsHF1i4xjjlsb_W1jT70IV0LaeCViUUR Q8/viewanalytics e em https://docs.google.com/spreadsheets/d/12D_BLe8rmhkJfjAJjRU7XenxY3Rjr2A1gY4CSLDYn8/edit?usp=sharing.

O tratamento dos dados (procedimento 5) foi realizado utilizando o Google Planilhas. Como algumas questões permitiam resposta descritiva (no campo "Outro"), as respostas deste campo foram classificadas nas demais alternativas existentes ou em categorias que não eram contempladas nas alternativas (neste caso se caracterizaria o conceito de "outro", de fato). Com base nesse tratamento, procedeuse à análise (procedimento 6), que é descrita na Seção 3.

\section{RESULTADOS E DISCUSSÕES}

Dado que parte dos resultados já foram discutidos na Seção 2, esta Seção apresentará em maiores detalhes os resultados e discussão sobre os questionários aplicados no IFC Campus Fraiburgo e nos demais Campi do IFC.

Sobre a pesquisa de opinião aplicada aos servidores do IFC Campus Fraiburgo, percebe-se que aqueles que já realizaram atividades de pesquisa sentem dificuldades devido ao excesso de burocracia e ausência de recursos financeiros para custear as despesas do projeto, tais como apresentação de trabalhos em eventos e compra de materiais. Também nota-se que há pouco tempo para realização de tais atividades e falta de interesse dos alunos. Precisa-se de mais capacitação para o coordenador e para os alunos. Ao referirem-se a demanda de alunos relatam que esta não existe, pelo fato de faltar projetos, bolsas e pelo desconhecimento dos projetos existentes. Já para o favorecimento da realização das atividades de pesquisa, percebe-se que os alunos tem potencial para realizar atividades tendo a oportunidade de resolver problemáticas da sociedade. Contudo, é preciso realizar um levantamento de demandas de pesquisa na região do Campus Fraiburgo. 
Em contrapartida, aqueles que não fizeram nenhum projeto de pesquisa relatam que nunca tentaram, pois veem que há um excesso de burocracia, falta de tempo para conciliar o trabalho com a criação de um projeto e reuniões para discussão do mesmo. Esclarecem que em determinadas vezes há interesse dos alunos, mas que não se propõem a fazer muitas vezes pela falta de capacitação e incentivo. Por outro lado, foi citado por vários entrevistados, que ao realizar atividades de pesquisa ocorre a qualificação tanto para o discente quanto para os servidores envolvidos, em busca de favorecer um grupo ou a comunidade da região.

A partir dessa análise, sugere-se algumas ações que poderiam ser realizadas visando tratar das problemáticas apresentadas: 1) incentivar mais os servidores; 2) aumentar o tempo para a realização das atividades de pesquisa; 3 ) disponibilizar mais bolsas estudantis com valores mais altos que os atualmente praticados; 4) diminuição da burocracia; 5) aumentar o número de vagas para realização de projetos; 6) ampliar os recursos para custeio do projeto e apresentação de trabalhos; e 7) buscar integração da instituição com a problemática local.

Sobre a pesquisa de opinião aplicada aos servidores de todos os Campi do IFC, esta obteve 151 respostas. A seguir é apresentada uma análise destes dados e algumas sugestões a respeito do que pode ser feito para tratar das problemáticas apresentadas. Os gráficos/figuras mencionados estão no Apêndice $E$, com vistas à facilitar a organização do texto.

Aproximadamente $2 / 3$ dos respondentes realizaram pesquisa e extensão. Ou seja, há demanda pelas duas modalidades e não há uma mais demandada do que a outra (questões 2 e 3 - Figuras 1 e 2). Observa-se que, também, a adoção a esse tipo de atividades mantém-se no último ano (questão 12 - Figura 11).

A burocracia (antes e durante a realização dos projetos) é apontada como um dos principais pontos a serem abordados para diminuir as dificuldades e favorecer a realização dos projetos (questões 4, 6, 7, 8 - Figuras 3, 5, 6, e 7, respectivamente). Com base nisso, avaliou-se o fluxo de ações necessárias para submissão do projeto, a saber:

1. Escrita do projeto;

2. Submissão para a Comissão de Avaliação de Projetos de Pesquisa (CAPP) e para o Comitê de Ética em Pesquisa com Seres Humanos - CEPSH (quando for o caso);

3. Cópia do parecer favorável da CAPP à submissão da proposta ao edital; e

4. Submissão via sistema acadêmico SIGAA dos seguintes documentos:

a. Formulário de Identificação do Projeto;

b. Projeto;

c. Currículo Lattes;

Após a aprovação da proposta, é preciso submeter os seguintes documentos:

1. Comprovante de matrícula do Bolsista;

2. Histórico escolar do Bolsista;

3. Cópia do RG, CPF e comprovante de endereço do Bolsista;

4. Declaração do orientador de que o bolsista atende aos requisitos do Edital; 
5. Declaração do orientador de que possui carga horária de trabalho disponível para desenvolvimento do projeto;

6. Declaração do Bolsista de que não possui vínculo empregatício ou acúmulo de bolsa estudantil;

7. Autorização dos pais do bolsista, quando menor de idade;

8. Termo de responsabilidade do orientador; e

9. Termo de sigilo e confidencialidade assinado por cada um dos membros do projeto. documentos:

Durante a execução da proposta, é preciso submeter os seguintes

1. Relatórios Mensais:

a. De atividades do projeto;

b. De cumprimento de horas de atividades de pesquisa pelo orientador;

2. Relatório Parcial; e

3. Relatório Final.

No processo realizado para submissão da proposta não são vislumbradas possíveis alterações. No processo após a aprovação da proposta, como não se pode prescindir da documentação solicitada, poderiam ser melhorados os seguintes pontos:

1. Itens 1,2 e 3 poderiam ser solicitados pela própria Coordenação de Pesquisa/Extensão ao Registro Acadêmico do Campus;

2. Unir em um único documento os itens 4,5 e 8;

3. O item 9 poderia ser um único documento, assinado por todos os componentes do projeto; e

4. Os itens 6 e 7 poderiam ser condensados em um único documento;

No processo que ocorre durante a realização do projeto, poderiam ser melhorados os seguintes pontos:

1. Percebe-se que os relatórios mensais tem foco principalmente no controle sobre a carga horária docente. Sugere-se fazer o acompanhamento mensal por meio de relatórios com foco nos objetivos educacionais do projeto;

2. Sobre o relatório parcial, há pouco foco sobre o que foi realizado no projeto até aquele momento. Assim, o relatório acaba sendo vago e inexpressivo. Sugerese extinguir este relatório e manter apenas os relatórios mensais, sendo o acompanhamento do projeto feito por meio destes; e

3. Relatório final poderia ser mantido na íntegra.

Ressalta-se que esses pontos que podem ser melhorados nos relatórios poderiam ajudar a resolver a falta de acompanhamento do projeto, apontada nas respostas à questão 4 (Figura 3).

Ainda sobre a questão 4 , aponta-se a falta de acompanhamento do projeto pela instituição. A previsão contida nas Resoluções do IFC sobre este aspecto é o acompanhamento via relatórios parcial e final, bem como mensalmente (sem mencionar como isso seria feito). Sugere-se, para resolver esta demanda, a 
proposição de cursos/encontros de formação no período do lançamento dos editais para os proponentes. Ao longo do ano, sugere-se a realização de encontros deste tipo para os projetos em execução.

Sobre a questão 5 (Figura 4 - fatores que favorecem/incentivam a realização de atividade de pesquisa/extensão), aponta-se a possibilidade de interação servidor/aluno com a comunidade externa. Neste ponto, percebe-se que a pesquisa e a extensão são vistas em uma relação dialógica com a sociedade e com os integrantes do processo pedagógico.

Sobre a questão 6 (Figura 5 - fatores que dificultam/desmotivam a realização de pesquisa/extensão), é apontada a falta de tempo para a realização do projeto. Em relação ao tempo para execução de projetos (por parte dos servidores), nas respostas da questão 5 (Figura 4) a existência de tempo é apontada como um fator que favorece a realização destas atividades, enquanto nas respostas da questão 6 (Figura 5) a falta de tempo é apontada como um fator que dificulta a realização de projetos. Nas respostas das questões 7 e 8 (Figuras 6 e 7, respectivamente) é sugerido, como fator que favoreceria a realização de atividades de pesquisa e de extensão, a possibilidade de cumprir a Carga Horária destinada a tais atividades fora do Campus.

Ainda em relação ao tempo para realização de projetos, as respostas para as duas últimas questões (Figuras 12 e 13) mostram que mais de $2 / 3$ dos respondentes utiliza, para pesquisa, até 3 h por semana. Para extensão o resultado é similar: aproximadamente $58 \%$ dos respondentes utiliza até $3 \mathrm{~h}$ por semana.

Para uma análise mais aprofundada destes resultados recomenda-se recorrer à Reitoria do IFC, a qual, segundo o conhecimento dos autores deste trabalho, recentemente realizou um estudo com vistas a analisar com quais atividades a carga horária de trabalho dos servidores era preenchida. docentes:

Para tratar desta questão, sugerem-se duas mudanças, ambas aplicáveis aos

1. Permitir que a carga horária de atividades de pesquisa e de extensão seja realizada fora do Campus (o que demanda estudo da legislação pertinente); e

2. No caso em que atividades administrativas estejam preenchendo a carga horária dos servidores de modo a limitar a realização de atividades de pesquisa e de extensão, diminuir estas atividades. Caso as atividades que estejam "lotando" a carga horária sejam as de ensino, recomenda-se diminuir a oferta de cursos de Formação Inicial e Continuada e focar na oferta de cursos regulares, permitindo assim que os docentes aloquem menos tempo para atividades de ensino e mais tempo para atividades de pesquisa e de extensão vinculadas aos cursos regulares.

Também, imagina-se que a formação continuada (para todos os servidores) tendo em vista a elucidação dos trâmites dos projetos no sistema acadêmico poderia reduzir a "sensação" de falta de tempo.

Em relação ao tempo dos discentes para participação em projetos, a partir da realidade do Campus Fraiburgo e considerando a perspectiva do Ensino Médio Integrado (EMI), percebe-se que já há movimentos buscando ampliar esse tempo. Em nossa visão, há a tendência de que haja certa saturação de atividades para os discentes, em função da quantidade de componentes curriculares (aproximadamente 20 em cada um dos 3 anos do curso). Neste sentido, há um movimento ocorrendo no 
IFC de formulação de Diretrizes para o EMI, que culminarão na reformulação destes cursos prevendo maior integração entre áreas do conhecimento e curricularização de pesquisa e de extensão, o que deve ampliar o tempo "fora de sala de aula" dos discentes. Além disso, em 2018, o Campus Fraiburgo experimentou uma organização do EMI que previa entre 8 e 11 períodos semanais de 45 minutos cada, dedicados a "atividades alternativas". Nestes períodos, os alunos poderiam escolher a quais atividades se dedicar, dentre as quais estavam os projetos de pesquisa e de extensão. A efetividade desta ação ainda precisa ser melhor analisada, mas partindo da experiência pessoal dos autores, foi possível levar a cabo os projetos com participação mais efetiva dos discentes.

A questão financeira também é apontada como um dos pontos importantes para a realização de atividades de pesquisa e de extensão. É apontada a falta de recursos para custeio de despesas dos projetos e para apresentação de trabalhos (questões 4, 6, 7 e 8 - Figuras 3, 5, 6 e 7, respectivamente). Também aponta-se, com menor ênfase, a quantidade de bolsas e o valor pago aos bolsistas como limitadores para a realização dos projetos.

Hoje, os Campi do IFC disponibilizam, pelo menos, $1 \%$ do orçamento de custeio para Pesquisa e 1\% para Extensão, montante que deve ser dividido entre bolsas estudantis e custeio dos projetos. No Campus Fraiburgo a realidade é essa, ou seja, são reservados $1 \%$ para cada departamento.

A Pró-Reitoria de Pesquisa e a Pró-Reitoria de Extensão do IFC (PROPI e PROEX) possuem editais majoritariamente de bolsas estudantis. Para apresentação de trabalhos, é possível utilizar de recurso disponibilizado pela Reitoria para esta finalidade. Contudo, o edital de 2017 foi cancelado e em 2018 não foi lançado edital. Ou, ainda, pode-se utilizar o recurso de capacitação de servidores disponibilizado pelo Campus (como já ocorre no Campus Fraiburgo). As bolsas são de $\mathrm{R} \$ 200,00$ para estudantes do Ensino Médio e de $\mathrm{R} \$ 400,00$ para estudantes de Nível Superior. Para tentar resolver essa problemática, sugere-se:

- Ampliar o recurso de capacitação de servidores nos Campi, com reserva para apresentação de trabalhos;

- Lançar editais de fomento a apresentação de trabalhos e publicação em periódicos, pela Reitoria;

- Ampliar os recursos que os Campi reservam para Pesquisa e Extensão, de 1\% para, pelo menos, $2 \%$ do orçamento, com previsão de reserva para custeio de despesas de projetos; e

- Ampliar as bolsas de ensino superior para $\mathrm{R} \$ 600,00$.

Também percebe-se a necessidade de estudar como podem ser equalizadas duas questões resultantes desta pesquisa: 0 aumento de bolsas estudantis e 0 aumento dos valores das bolsas, pontos que parecem divergentes à princípio.

Sobre a interação dos servidores com as problemáticas locais, vale ressaltar que há previsão (e de certo modo, incentivo) legal para que esta aproximação aconteça. As respostas para a questão 5 (Figura 4) apontam que a interação com a sociedade é um fator que favorece a realização de projetos. As respostas às questões 7 e 8 (Figuras 6 e 7, respectivamente) corroboram esta visão: aponta-se que a busca de integração da instituição com as problemáticas locais é uma ação institucional que poderia favorecer a realização de projetos de pesquisa e de extensão. 
A sugestão para este tópico é de que os coordenadores de pesquisa e de extensão possam comprometer-se em visitar instituições públicas de sua região para buscar possíveis parcerias/projetos.

A necessidade de capacitação/apoio institucional para escrita/execução de projetos fica clara a partir das respostas das questões 4, 6, 7 e 8 (Figuras 3, 5, 6 e 7, respectivamente). A partir da realidade do Campus Fraiburgo (o que reflete ações deste Campus e, em parte, da Reitoria), percebe-se que, de fato, não são realizados treinamentos/capacitações sobre a escrita de projetos nem sobre processos envolvidos na submissão/execução destes. A sugestão, neste caso, é que os Campi realizem treinamentos/capacitações sobre submissão de projetos via sistema SIGAA e, também, para a escrita de projetos.

\section{CONCLUSÕES E CONSIDERAÇÕES FINAIS}

A partir do exposto, é possível afirmar que os objetivos deste trabalho foram atingidos, a saber: 1) Buscar parcerias entre o IFC e instituições públicas e privadas nas áreas de pesquisa, desenvolvimento tecnológico e extensão; 2) Propor iniciativas que tenham a perspectiva de integrar ensino, pesquisa e extensão; e 3)Entender como melhor fomentar as atividades de pesquisa e extensão do IFC Campus Fraiburgo .

Apesar de atingidos, percebe-se a necessidade de aprofundar a análise com vistas a entender a realidade de cada Campus e cada arranjo produtivo local e adaptar as sugestões propostas a cada um dos Campi. Com isso, queremos dizer que uma solução não servirá para todos, apesar da problemática muitas vezes ser a mesma. Percebe-se, ainda, a oportunidade de analisar as sugestões apresentadas e, na medida do que for possível, aplicá-las na tentativa de resolver as problemáticas abordadas neste trabalho. Daí surge a oportunidade de analisar se as sugestões surtiram o efeito esperado ou se precisam de adaptações.

Ao final deste trabalho, percebe-se que o olhar crítico sobre as atividades do dia-a-dia e o "jeito de trabalhar" foi proveitoso, permitindo perceber alguns pontos que podem melhorar e outros que precisam manter-se como são. Neste sentido, este trabalho buscou também criticar as ações da gestão educacional em pesquisa e extensão e entender o que leva a gestão a trabalhar desta ou daquela maneira e, nesta seara, sempre é possível enxergar um ponto a melhorar.

Por fim, entendemos que este trabalho também teve como contribuição ser o "pontapé inicial" de um processo de revisão/renovação das ações da gestão educacional em pesquisa e extensão do IFC Campus Fraiburgo, com vistas a incentivar a realização de projetos nestas áreas e a maior integração com arranjos produtivos locais. Sem dúvida, há espaço para realização de estudos mais aprofundados que podem tomar estes resultados como ponto de partida.

\section{REFERÊNCIAS}

FRAIBURGO, Câmara Municipal de. Projeto de Lei № 2589/2019 é aprovado na Câmara. 2019. Disponível em: $<$ https://www.camarafraiburgo.sc.gov.br/camara/conteudo/noticias/Noticias/1/2019/8 45ㄱ. Acesso em: 10 out. 2019. 
FREITAS JUNIOR, Vanderlei; CARVALHO, Wenis Vargas de. Os desafios para a consolidação da pesquisa aplicada no Instituto Federal Catarinense: uma análise do câmpus avançado Sombrio. Metodologias e Aprendizado, v. 2, n. 2, p. 63, 2020. Disponível em: https://publicacoes.ifc.edu.br/index.php/metapre/article/view/1339. Acesso em: 30 jun. 2020.

INSTITUTO FEDERAL CATARINENSE. Edital no 040/2017 - DG/IFC - Campus Fraiburgo, de 11 de dezembro de 2017: Apresentação de projetos integrados de pesquisa e extensão e seleção de estudantes do ensino médio técnico como bolsistas do Instituto Federal Catarinense. - Campus Fraiburgo. Disponível em: $<$ http://editais.fraiburgo.ifc.edu.br/?p=1064>. Acesso em: 18 dez. 2018.

MARQUES, Maristela Beck; VIEIRA, Josimar de Aparecido. Indissociabilidade do ensino, pesquisa e extensão na prática profissional do ensino médio integrado à educação profissional. Revista de Educação, Ciência e Tecnologia do IFRS, v. 7, n. 1, p. 187, 2020. Disponível em: https://periodicos.ifrs.edu.br/index.php/ScientiaTec/article/view/4131. Acesso em: 06 jun. 2020. 